OPEN

SUBJECT AREAS:

BIOPOLYMERS

MESENCHYMAL STEM CELLS

Received

21 January 2014

Accepted

7 July 2014

Published

28 July 2014

Correspondence and requests for materials should be addressed to W.L. (wgliu@tju.edu.

$\mathrm{cn})$

* These authors contributed equally to this work.

\section{Controlled Heterogeneous Stem Cell Differentiation on a Shape Memory Hydrogel Surface}

\author{
Yanjiao Han*, Tao Bai* \& Wenguang Liu
}

Collaborative Innovation Center of Chemical Science and Engineering(Tianjin), Tianjin 300072, P. R. China.

The success of stem cell therapies is highly dependent on the ability to control their programmed differentiation. So far, it is commonly believed that the differentiation behavior of stem cells is supposed to be identical when they are cultured on the same homogeneous platform. However, in this report, we show that this is not always true. By utilizing a double-ion-triggered shape memory effect, the pre-seeded hMSCs were controllably located in different growth positions. Here, we demonstrate for the first time that the differentiation behavior of hMSCs is highly sensitive to their growth position on a hydrogel scaffold. This work will not only enrich the mechanisms for controlling the differentiation of stem cells, but also offer a one-of-a-kind platform to achieve a heterogeneously differentiated stem cell-seeded hydrogel scaffold for complex biological applications.

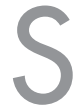

tem cells can differentiate into various cell lineages with different functions, thus holding great potential in tissue engineering and regenerative medicin $e^{1,2}$. Recent studies have shown that the fate of stem cells can be controlled by both chemical ${ }^{3}$ and physical ${ }^{4}$ factors $^{3-5}$. For the biochemical factors, it is well-established that several chemicals including hormones, growth factors and small molecules can modulate the biological activity of stem cells and direct their differentiation down specific pathways ${ }^{3,6}$. On the other hand, the physical properties $^{4,5-10}$ including stiffness ${ }^{11,12}$, porosity ${ }^{13,14}$, surface pattern ${ }^{15,16}$ and geometry s $^{17,18}$ of the extracellular matrix have been recognized as crucial factors in regulating the differentiation of stem cells thus far. For instance, human mesenchymal stem cells (hMSCs) are shown to specify lineage and acquire phenotypes with extreme sensitivity to the elasticity of the substrate ${ }^{11,1}$. A deeper analysis reveals that the stem cells are able to sense the mechanical property of their surrounding environment via integrin binding and initiate the specific differentiation process $^{20-22}$.

In the transition from fundamental studies to clinically relevant applications of stem cells, it is very important to elucidate the behavior of stem cells in complex geometrical scaffolds in which the stem cells are located in different positions and supported by the platform in different ways. However, the influence of the growth position on the differentiation behavior of stem cells is unknown, but of great implication to understanding regenerative phenomena as well as directing design of tissue engineering scaffolds. It is generally difficult to achieve various culture positions for stem cells on the same platform since normal 2D cultures only enable the cells to grow on the top of platforms. In our previous work $\mathrm{k}^{23-25}$, we demonstrated polyacrylonitrile (PAN)-based copolymer hydrogels were able to exhibit a shape memory effect by manipulating the reversible formation of dipole-dipole interactions in response to zinc ions. Furthermore, the self-rolling/folding and arbitrary 3-D shape changes could be achieved and well maintained if the gel was pre-programmed with a surface pattern. The goal of this work was to explore the differentiation behavior of hMSCs when they were grown on different places of a single piece hydrogel. The hMSCs were cultured on the surface of PAN based hydrogels, while their growth location was altered by a shape memory effect. Finally, the hydrogel-hMSC constructs were cultured in a bipotential differentiation medium (osteogenic and adipogenic). As a result, we were able to examine the direct influence growth location could have on the fate choice of hMSCs. This work demonstrates that the location of a scaffold where stem cells grow may be used to control complicated cellular functions such as stem cell differentiation. These results are not only fundamentally interesting, but also could lead to simple and controllable scaffold formulations for the application of hMSC-based regenerative medicine. 

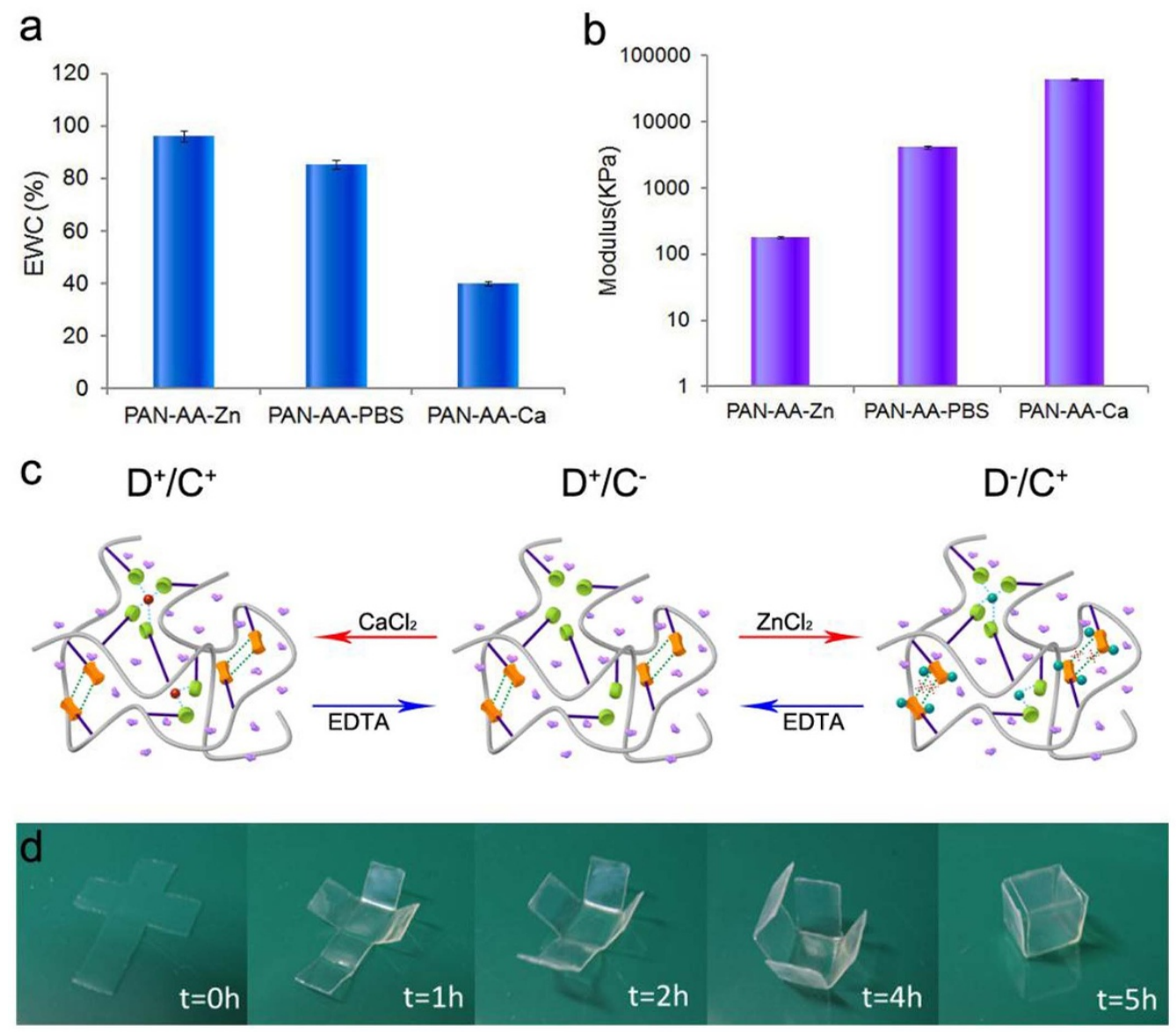

Figure 1 1 EWC (a) and modulus (b) of AN-AA-Zn, AN-AA-PBS and AN-AA-Ca hydrogels. (c) A schematic diagram delineating the multiple association and dissociation in PAN-based hydrogels. The hydrogel can selectively stay in three different states. i.e. The $\mathrm{D}^{+} / \mathrm{C}^{+}$, the $\mathrm{D}^{+} / \mathrm{C}^{-}$and the $\mathrm{D}^{-} / \mathrm{C}^{+}$states. The red spheres denote calcium ions and the cyan spheres denote zinc ions. (d) Real-time observation of the recovery of the box-shaped hydrogel.

\section{Results}

Double-ion controlled triple shape memory. In this study, on the basis of the $\mathrm{Zn}^{2+}$ responsive behavior of PAN hydrogel reported in our previous work ${ }^{23}$, the hydrogel was further incorporated with carboxyl groups which were used to fix the temporary shape of the hydrogel due to their coordination with calcium ions ${ }^{26}$. We copolymerized monomers of acrylonitrile (AN), acrylic acid (AA) and a biodegradable crosslinker, $\mathrm{N}, \mathrm{N}^{\prime}$-bis(acryloyl)cystamine (BAC), to form dipole-dipole reinforced (DDR) hydrogels whose molecular structure is depicted in Scheme S1. Similar to what we reported previously ${ }^{23}$, due to the reinforcement from strong noncovalent dipole-dipole interactions among cyano groups, the poly(acrylonitrile) (PAN) hydrogels presented here exhibited 1.5 MPa tensile strengths, $160 \%$ elongation at break, and $9.3 \mathrm{MPa}$ compressive stresses at $80 \%$ strain, with equilibrated water contents ranging from $78 \%$ to $83 \%$ (Supplementary Fig. S2 and Table S1). In order to avoid stiffness-favored differentiation of stem cells as reported $^{27}$, the moduli of all selected samples shown in Table S1 are beyond the normal tissue-level which may specifically induce osteogenesis or adipogenesis. Besides, the gels are degradable in a redox environment (Supplementary Fig. S3).

We began to investigate the influence of divalent ions on the presented hydrogels. Here, $\mathrm{Zn}^{2+}$ and $\mathrm{Ca}^{2+}$ were selected since they were relatively biocompatible and can selectively bind to $\mathrm{CN}$ and $\mathrm{COO}^{-}$ groups under certain circumstances. We first tested the influence of $\mathrm{Ca}^{2+}$ ions on the equilibrium water content (EWC) of the AN-AAPBS hydrogel (equilibrated in PBS). The hydrogel samples were equilibrated in either $3.7 \mathrm{M} \mathrm{ZnCl}_{2}$ (denoted as $\mathrm{AN}-\mathrm{AA}-\mathrm{Zn}$ ) or $5 \mathrm{mM} \mathrm{CaCl}_{2}$ (denoted as AN-AA-Ca) solution for at least three days prior to testing. Since $\mathrm{Ca}^{2+}$ can serve as chelation center among carboxyl groups and further densify the hydrogel network, the EWC of AN-AA-Ca is about 50\% lower than AN-AA-PBS (Fig. 1a). Conversely, for AN-AA-Zn hydrogels, $\mathrm{Zn}^{2+}$ can selectively disassociate dipole-dipole interactions and thus significantly loosens the hydrogel network. As presented in Fig. 1a, the EWC of AN-AA$\mathrm{Zn}(96.7 \%)$ is $10 \%$ higher than that of AN-AA-PBS. Additionally, the influence of divalent ions on the moduli of selected hydrogels was observed. When dipole-dipole interactions were disassociated by $\mathrm{Zn}^{2+}$, the modulus of the selected hydrogel decreased sharply to $179 \mathrm{KPa}$ (Fig. 1b). Also, we found that the strong chelation formed among carboxyl groups and $\mathrm{Ca}^{2+}$ was able to significantly enhance the modulus of the selected hydrogel to $9.53 \mathrm{MPa}$ (Fig. 1b). It should be noted that both dipole-dipole and $\mathrm{Ca}^{2+}$ ion reinforcement can be reversibly built-in or removed from the system. The shape memory efficiency of AN-AA-PBS hydrogel was characterized by an anglecontrolled experiment. First, we put one straight strip AN-AA-PBS hydrogel into a $3.7 \mathrm{M} \mathrm{ZnCl}_{2}$ solution. After reaching adsorption equilibrium, the AN-AA-Zn hydrogel was bent into a " $U$ " shape with the angle between the two arms being fixed at 180 degrees. $0.01 \mathrm{mM}$ EDTA solution $(\mathrm{pH}=7.4)$ was then used to remove the divalent ions from the hydrogel network. As a result, the AN-AAPBS hydrogel was transformed from $\mathrm{D}^{-} / \mathrm{C}^{+}$to $\mathrm{D}^{+} / \mathrm{C}^{-}$state. In a following step, the straight strip shape could be recovered again by equilibrating the AN-AA-PBS hydrogel in a $3.7 \mathrm{M} \mathrm{ZnCl}_{2}$ solution 

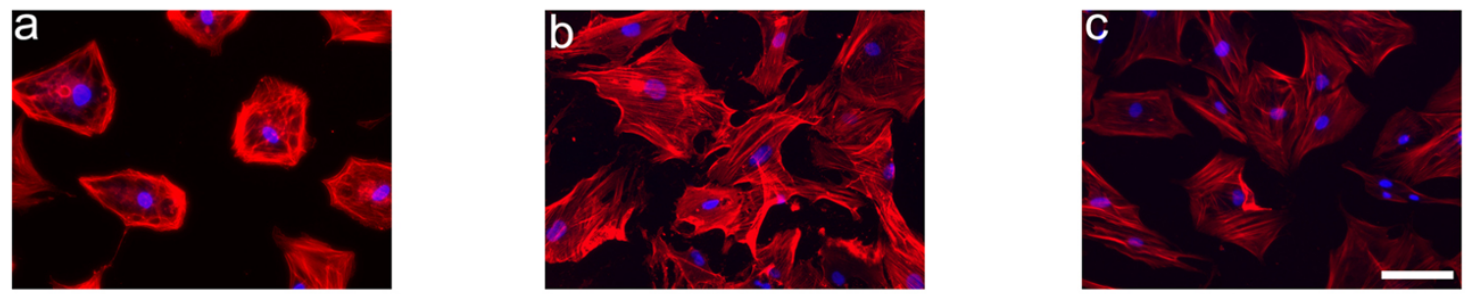

d

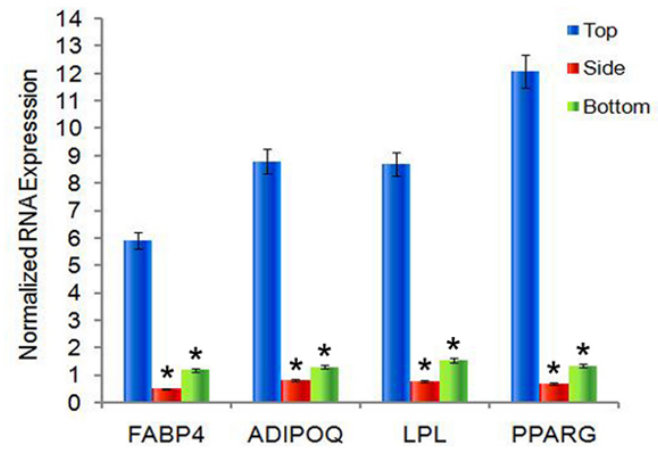

e

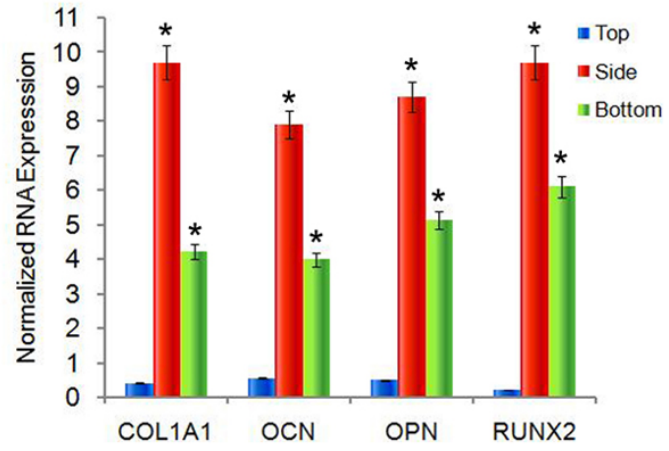

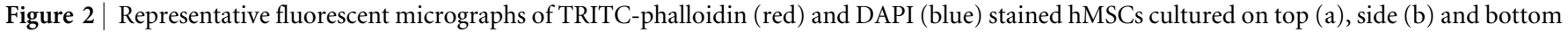

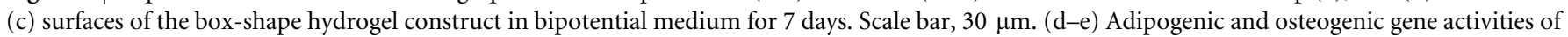

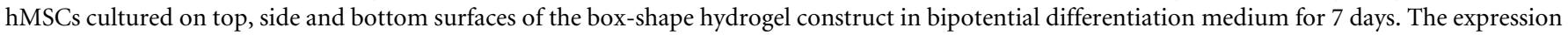

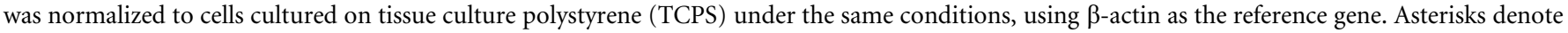
statistical significance compared with top surfaces ( ${ }^{*} p<0.001$, ANOVA and Tukey test). Error bars represent standard error of the mean from 5 individual experiments.

again (Phase I). Figure S4a shows that this shape memory effect (Phase I) can be repeated for another two times with selectively associating/disassociating the dipole-dipole interaction.

We further demonstrated the role of $\mathrm{Ca}^{2+}$ coordination in achieving the shape memory effect. Herein, the above hydrogel after the Phase I experiments was continued to be used for exhibiting the shape-memory effect resulting from divalent ion coordination (Phase II). After bending AN-AA-PBS hydrogel into a " $U$ " shape as demonstrated above, the U-shape AN-AA-PBS gel was transferred into a $5 \mathrm{mM} \mathrm{CaCl}_{2}$ solution. After reaching equilibrium in $\mathrm{CaCl}_{2}$ solution, this U-shape can be $100 \%$ fixed (Table S1). The U-shape can also recover to the original state after immersing the gel into EDTA $(0.01 \mathrm{mM})$ solution. Similar to the shape memory effect resulting from dipole-dipole interactions, this shape-memory effect from $\mathrm{Ca}^{2+}$ coordination can also be cycled (Fig. S4a). In the process of the angle-controlled experiment, we also measured the mechanical property of selected samples after each swelling-deswelling cycle. As presented in Figure S4b, the cyclic process only resulted in a slight change in the modulus of selected samples and we believed that this process should be completely reversible. Therefore, as illustrated in Fig. 1c, a DDR hydrogel can be selectively controlled to stay in three different states, i.e. $\mathrm{D}^{-} / \mathrm{C}^{+}$(without dipole-dipole reinforcement and with chelation), $\mathrm{D}^{+} / \mathrm{C}^{-}$(with dipole-dipole reinforcement and without chelation), and $\mathrm{D}^{+} / \mathrm{C}^{+}$(with both dipole-dipole reinforcement and $\mathrm{Ca}^{2+}$ chelation).

Achieving heterogeneous distribution of hMSCs via a shape memory effect. Based on our discussion above, it is expected that selectively switching between $\mathrm{D}^{+} / \mathrm{C}^{-}$and $\mathrm{D}^{-} / \mathrm{C}^{+}$states or between $\mathrm{D}^{+} / \mathrm{C}^{+}$and $\mathrm{D}^{+} / \mathrm{C}^{-}$states could realize a triple shape memory effect. In the Fig. 1d, an as-prepared hydrogel was cut into a cross-shaped sheet and purified by dialysis in PBS ( $\mathrm{pH}=7.4)$ for three days. The hydrogel was then transferred into a $3.7 \mathrm{M} \mathrm{ZnCl}_{2}$ solution to disassociate the dipole-dipole interactions. At the state of $\mathrm{D}^{-} / \mathrm{C}^{+}$, the gel became soft and able to readily fold into a cubic box (temporary shape 1). Subsequently, the hydrogel was converted into the $\mathrm{D}^{+} / \mathrm{C}^{-}$state by removing $\mathrm{Zn}^{2+}$ with an EDTA solution $(\mathrm{pH}=7.4,0.01 \mathrm{mM})$. As a result, the box shape was fixed by the re-formation of dipole-dipole interactions. The box-shape hydrogel was further unfolded into a flat shape (temporary shape 2) again and fixed in $5 \mathrm{mM} \mathrm{CaCl}_{2}\left(\mathrm{D}^{+} / \mathrm{C}^{+}\right)$. The hydrogel with temporary shape 2 was transferred into a basal medium. After equilibrium in the basal medium and being coated with fibronectin, hMSCs were seeded on the surface of the flat hydrogel. After 24 hours, unattached cells were washed away by refreshing the medium and the cell-seeded construct was folded automatically $\left(\mathrm{D}^{+} / \mathrm{C}^{-}\right.$state) by removing $\mathrm{Ca}^{2+}$ with EDTA-containing basal medium $(0.01 \mathrm{mM})$. The whole process was completed in five hours. Consequently, the hMSCs were homogeneously seeded on the 6 sides (Detailed characterization can be found in Supplementary Fig. S5, 6). We noted that the EDTA treatment did not cause the detachment of hMSCs.

Morphology and gene activity of hMSCs on each side. To verify if the growth position (top, side and bottom surface) of hMSCs influences the differentiation of hMSCs, the cell-hydrogel construct was transferred into a bipotential differentiation medium whose formulation was designed for inducing both adipogenesis and osteogenesis. After a 7-day differentiation process which is widely used $^{18,20}$, the cell-hydrogel construct was cut into 6 different pieces according to their position. The morphology of the hMSCs was visualized by immunostaining F-actin and nuclei. It was clear that after the 7-day differentiation, hMSCs attained significantly different morphologies on different sides (Fig. 2a-c). Hanging from the top surface, most hMSCs formed a round shape (Fig. 2a). In contrast, the hMSCs on the side and bottom surface were prone to assume an outstretched shape (Fig. 2b-c). As reported previously ${ }^{10,18}$, the shape of hMSCs is able to modulate cell traction and thus directs them to differentiate into different lineages. The round-shape hMSCs are inclined to differentiate down the adipogenic pathway, while outstretched-shape hMSCs are inclined to differentiate down 
the osteogenic pathway. Herein, we characterized the heterogeneous differentiation by RNA expression and immunocytochemistry (ICC) assays.

We first tested the differentiation behavior of the hMSCs on the cell-hydrogel construct based on their RNA expression. RNAs were isolated according to vendor instructions and quantitative real-time PCR (qRT-PCR) was performed to determine the expression of characteristic adipocyte and osteocyte genes. In the Fig. $2 \mathrm{~d}-\mathrm{e}$, the expression was normalized to cells cultured on tissue culture polystyrene (TCPS) under the same conditions, using $\beta$-actin as the reference gene. Among the expression of a panel of adipogenic genes from hMSCs on top, side and bottom surfaces, peroxisome proliferatoractivated receptor gamma (PPARG) is a crucial regulator of adipogenesis and highly related to the uptake and storage of lipids. Fatty acid binding protein 4 (FABP4) is a carrier protein for fatty acids that is mainly expressed in adipocytes. Adiponectin, which regulates fatty acid breakdown, is encoded by the ADIPOQ gene in the human body. Lipoprotein lipase (LPL) is an enzyme that can hydrolyze triglycerides in lipoproteins. As presented in Fig. 2d, all of these four adipogenic biomarkers were expressed at significantly higher levels in the hMSCs from the top compared with those on the side or the bottom. At the same time, one can find that the hMSCs on the bottom expressed a comparable level of adipogenic biomarkers to the hMSCs on TCPS while their expression is slightly restrained when they were grown on the side surfaces.

We also measured the expression of osteogenic genes from hMSCs on the top, side and bottom surfaces (Fig. 2e). Runt-related transcription factor 2 (RUNX2) is a key transcription factor essential for osteogenic differentiation. COL1A1 (Collagen, type I, alpha 1) is human gene that encodes the major component of collagen I, which can be found in osteoprogenitor cells. Osteocalcin $(\mathrm{OCN})$ is one kind of noncollagenous protein secreted exclusively by osteoblasts. Osteopontin (OPN) is expressed predominantly by osteoblasts, osteocyctes and osteoclasts and has been implicated as an important factor in bone remodeling. In contrast to our finding on the expression of adipogenic biomarkers, the expression of these osteogenic biomarkers was restrained when the hMSCs were grown on the top surface. It is noteworthy that hMSCs on the side surface demonstrate significantly higher expression of osteogenic biomarkers, while hMSCs on the bottom surface present lower expression. The above-mentioned RNA expression assay demonstrates that growth position could significantly influence the differentiation direction of cultured hMSCs. Compared with hMSCs under normal growth conditions, the "up-side-down" growth condition promotes the process of adipogenesis while the "wall-hanging" growth condition promotes the process of osteogenesis.

Histological staining for differentiated hMSCs on each side. We further aimed to explore certain chemicals and enzymes expressed within specifically differentiated hMSCs. Osteogenesis can be reflected by alkaline phosphatase (ALP), stained with Fast Blue salts. Likewise, adipogenesis in hMSCs can be demonstrated by the presence of lipids, visualized by staining with Oil Red O. We conducted a histological staining for both lipid and ALP in hMSCs cultured in the three positions. Staining for nuclei confirmed that the lipid droplets corresponded to single cells rather than clusters (Supplementary Fig. S8). As presented in Fig. 3a-c, stem cells differentiated positively on all surfaces. While nearly all the differentiated cells committed adipogenesis on the top, osteogenesis dominated on the side and bottom. It is also observed that hMSCs on the bottom are more prone to choose the osteogenic pathway rather than the adipogenic pathway. The quantification of the heterogenic differentiation is summarized in Fig. 3d. In addition, we quantified the calcium deposition with alizarin red staining in order to confirm the osteogenic differentiation. As presented in the Supplementary Fig. S9, the results were consistent to the ALP staining observed above.

ICC assay for hMSCs on hydrogel surfaces. The characteristic antigens on differentiated hMSCs were visualized via ICC assay. Herein, PPARG was used as a biomarker to visualize adipogenesis and RUNX2 was used as a biomarker to observe osteogenesis. Nuclei were stained by DAPI. Again, the hydrogel-cell construct was cut into different parts according to the position (i.e. top, side and bottom surface). In Fig. 4, it is observed that the hMSCs expressed PPARG more robustly when they were cultured on the top. In contrast, RUNX2 was weakly expressed on the top, indicating the osteogenesis was greatly inhibited due to the competition of adipogenesis. Meanwhile, one can find a reverse scenario when examining these biomarker expressions from the hMSCs on the side surface. When the MSCs are cultured on the side surface, expression of the osteogenic biomarker was much more pronounced compared to that
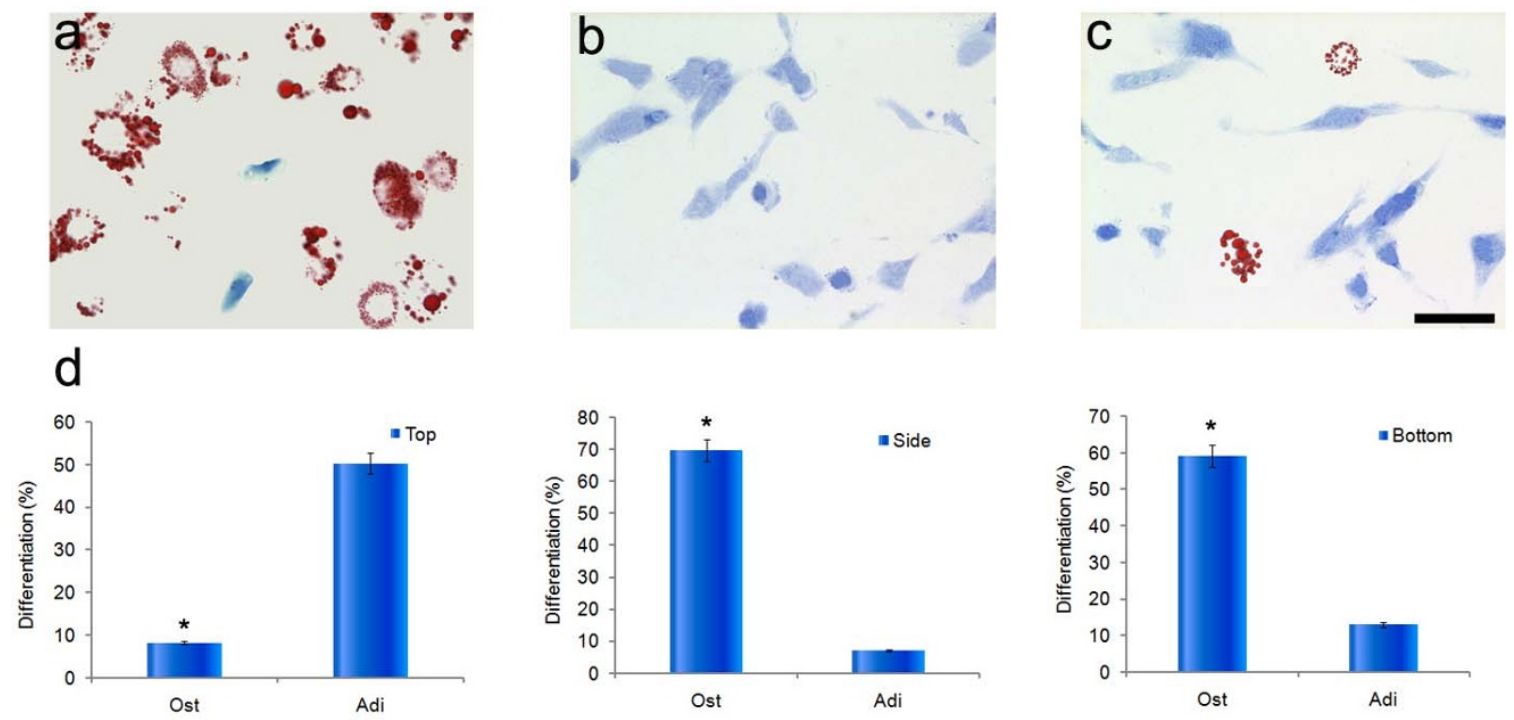

Figure 3 Representative bright-field images of histologically stained hMSCs on the top (a), side (b) and bottom (c) following 7-day bipotential differentiation medium incubation. Scale bar, $50 \mu \mathrm{m}$. (d) Percentage differentiation of hMSCs on different sides of cubic AN-AA-PBS hydrogels. Asterisks denote statistical significance compared with top surfaces $(* * \mathrm{p}<0.001, \mathrm{t}$-test). Error bars represent standard error of the mean from 5 individual experiments. 


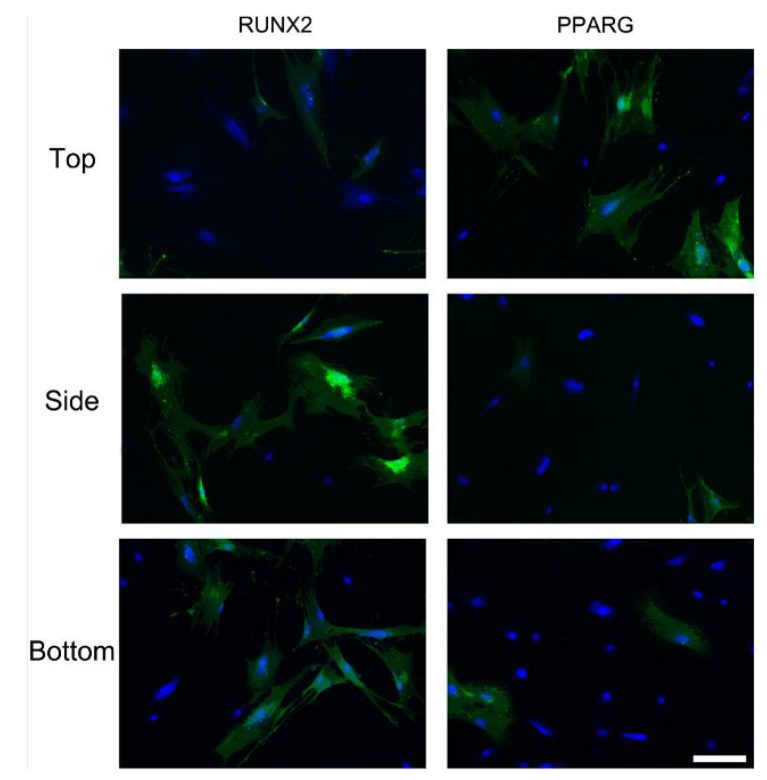

Figure 4 After 7 days of culture in bipotential differentiation medium, cell-hydrogel constructs of different positions (Top, Side and Bottom surface) were immunostained for adipogenic (PPARG) and osteogenic (RUNX2) biomarkers. Scale bar, $30 \mu \mathrm{m}$.

of adipogenic biomarker PPARG (Fig. 4). Comparatively, osteogenesis dominated the differentiation behavior of hMSCs on the bottom surface. The above results clearly demonstrate the growth position has a strong influence on the differentiation behavior of hMSCs under normal culture conditions.

Modulating differentiation by cytoskeletal manipulation. It has been proved that the differentiation of hMSCs depends on the cytoskeletal contractility ${ }^{7}$. In this work, we further tested the influence of several pharmacological agents known to modulate the cytoskeleton on the differentiation behavior of attached hMSCs on top, side and bottom via histological staining as mentioned above. The inhibitors were added into the media when the cells were seeded on the top of AN-AA-PBS hydrogel. The concentration of the added inhibitors were same in both basal and differentiation media. A 10day culture ( 3 days in a basal media and 7 days in a bipotential media) was employed before the histological staining. The percentage of hMSCs differentiating to osteoblasts or adipocytes in media without the addition of pharmacological agents is shown in Fig. 5a. Nocodazole is able to interfere with the polymerization of microtubules, thus increasing the cytoskeletal contractility. As seen in Fig. 5b, the drug indeed reversed the fate choice of hMSCs cultured on top and gave a high rate of osteogenesis in all cases. Three other drugs that can directly inhibit contractility of the cytoskeleton-Y27632, which inhibits ROCK, Cytochalasin D, which inhibits F-actin polymerization, and blebbistatin, which inhibits myosin II-resulted in a significant decrease in osteogenesis with a corresponding increase in adipogenesis, with the cultured condition barely having influence on differentiation (Fig. $5 c-e$ ). $\alpha 5 \beta 1$ integrin can mediate coupling of the cytoskeleton to the extracellular matrix prior to adhesion. Here, we examined the cells on different surfaces in the presence of function-blocking antibodies against $\alpha 5 \beta 1$ integrin. We found most hMSCs committed to adipogenesis in the presence of these antibodies, and the influence of mechanical property on differentiation was removed (Fig. 5f). These results are consistent with the role of cytoskeletal contractility in directing the fate in cells and further demonstrate the hMSCs can alter their cytoskeletal contractility when they are cultured on different surfaces of the cubic hydrogel. Representative images of the histological staining can be found in Supplementary Fig. S9.

\section{Discussion}

Current studies on stem cells are mostly focused on their behaviors on the top of $2 \mathrm{D}$ platforms or within $3 \mathrm{D}$ hydrogels $\mathrm{s}^{7,828-34}$. While some experimental techniques like hanging drop method has interesting implications that the growth position may influence cellular functions of stem cells, it is still not clear whether the specific fate of stem cells is able to be harnessed by their culture position $^{35}$. At the same time, it is highly desirable to achieve heterogeneous differentiation of stem cells on/in a single piece scaffold for many clinically relevant applications such as the construction of artificial organs. However, it is generally difficult to implement heterogeneous biophysical or biochemical cues in one homogeneous scaffold.

In this study, we developed a novel double-ion-triggered shape memory hydrogel. Introducing and removing two types of divalent ions could contribute to reversible dissociation of dipole-dipole pairings and divalent ion-chelation, thereby giving rise to a multiple shape memory effect. One remarkable feature of the reported hydrogel is its capability of automatic shape-changing, eliminating the risk of contaminating cells via manual manipulation. This shape memory effect could specifically modulate the differentiation behavior of stem cells via manipulation of the cytoskeletal contractility. During the fixation of a temporary shape by dipole-dipole interaction and calcium-chelation, hMSCs were seeded on the hydrogel surface; after extracting $\mathrm{Ca}^{2+}$, the hydrogel-hMSCs construct could automatically reshape into a cubic box where hMSCs were shown to exhibit heterogeneous differentiation behavior at different growth positions. The "up-side-down" growth position promotes the process of adipogenesis while the "wall-hanging" growth condition promotes the process of osteogenesis.

One hypothetic mechanism underlying this work could be the microgravity-induced organization of cytoskeleton ${ }^{36}$. The gravity of cells is generally overlooked since the weight of a cell in medium can only exert a small force of around $0.4 \mathrm{pN}^{37}$. However, relevant findings reveal that the cytoskeleton could act as an antenna for mechanical force and amplify these small forces ${ }^{38}$. As a result, the forces in the actin cytoskeleton alone are over 1 million times than the weight of cells ${ }^{37}$. In this work, the gravity of cells was counterbalanced by the supporting force when they were grown on the bottom and the inner cytoskeletal tension drove the most hMSCs to adopt osteogenic differentiation. The cells hanging upside down on the top of the platform were not supported and its own gravity could inhibit the cell from forming a stretched shape by downward pulling force, thus reducing the cytoskeletal tension. In contrast to that, the cytoskeletal tension could be amplified due to the gravitytraction induced stretched shape when the cells were grown on the side surface. Cell contraction regulated by biofactors ${ }^{39}$, signal pathways ${ }^{40}$ and substrate stiffness ${ }^{41}$ have been reported previously. In this work, we, for the first time, observed the distinct contraction behaviors of cells at different growth sites of a 3-D scaffold. Therefore, it is expected that the cytoskeletal contraction in cells grown on different sides of our hydrogels could be dramatically different. The involvement of additional cytoskeletal-disrupting agents further confirms our speculation that the different cytoskeletal contraction in hMSCs on different sides could dramatically influence their differentiation behavior. This is consistent to a previous model reported by Chen and others ${ }^{10,27}$. For example, nocodazole increases the contractility of the cytoskeleton and was able to drive the majority of hMSCs on all surfaces towards osteogenic differentiation. In contrast, cells on all surfaces exposed to molecules (Cytochalasin D, Blebbistatin and Y27632) that inhibit contractility were prone to differentiate into adipocytes. Furthermore, we could reduce the tension exerted by the cell on all surfaces with a corresponding inhibition of osteogenesis by blocking a portion of cell surface integrin receptor with an antibody. These results suggest the heterogeneous distribution of hMSCs by the reported shape memory effect could delicately modulate the cyto- 
a
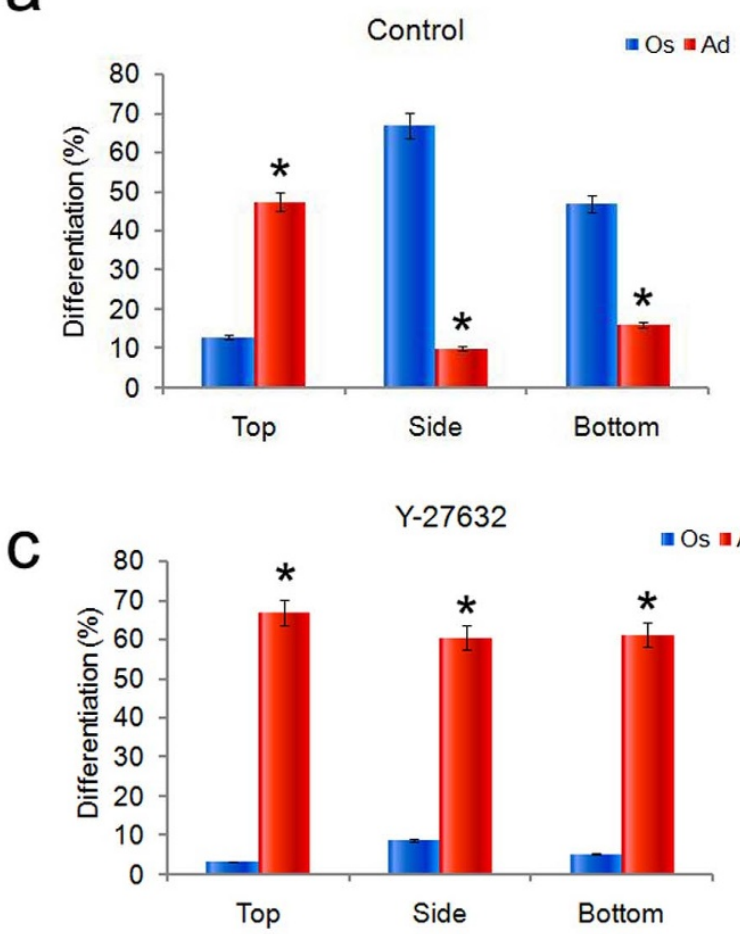

e

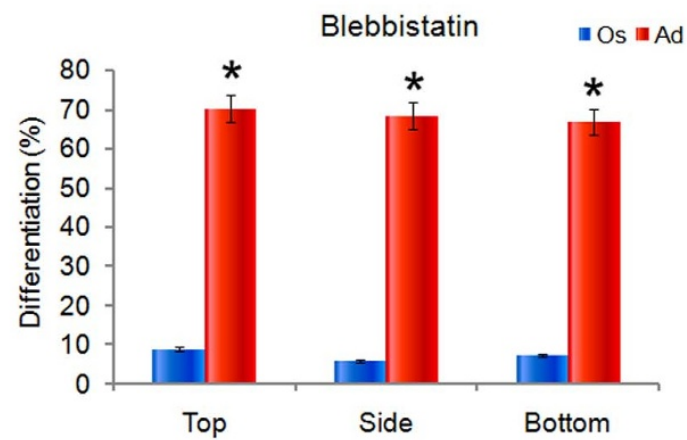

b
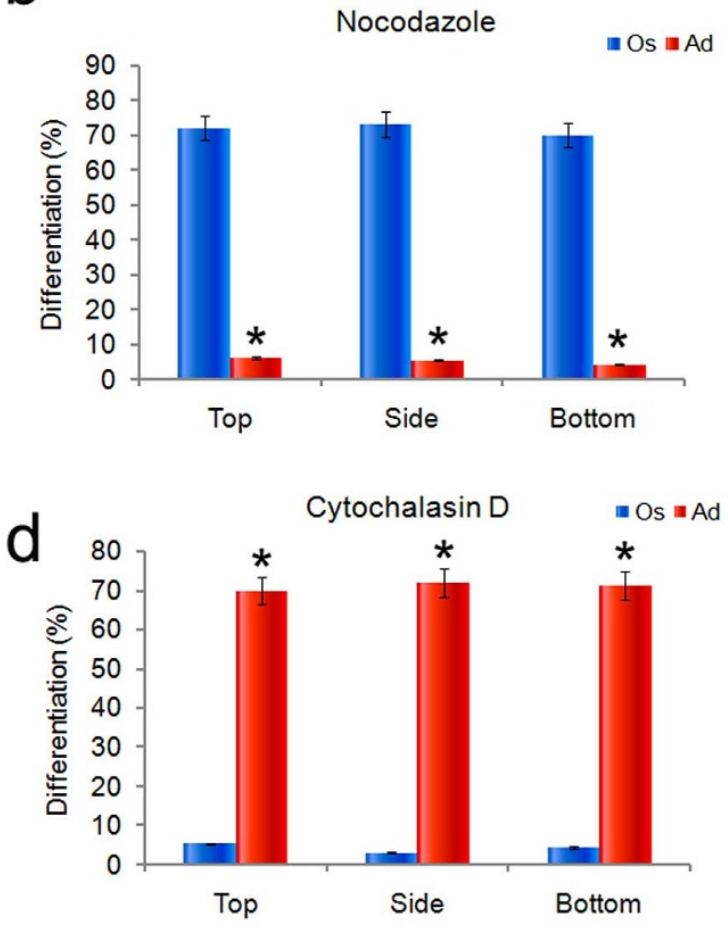

f

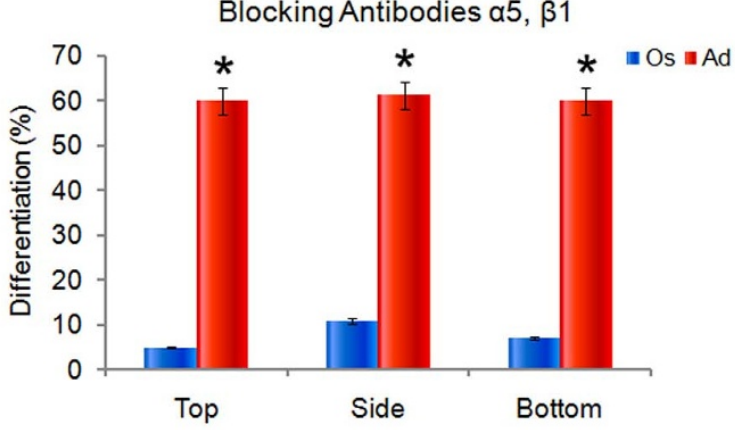

Figure 5 Percentage differentiation of hMSCs on different sides of cubic AN-AA-PBS hydrogels differentiating to adipocytes (stained by Oil red O) or osteoblasts (stained by Fast Blue salt) in the absence (a) and presence of cytoskeleton disruptors nocodazole (b), Y-27632 (c), cytochalasin D (d), blebbistatin (e) or integrin blocking antibodies $\alpha 5, \beta 1$ (f). As denoted by the asterisks, for all histological staining, the percent differentiation between adipogenesis and osteogenesis was significantly different among hMSCs on top, side and bottom surfaces $\left({ }^{*} p<0.001, t\right.$-test $)$. Error bars represent standard error of the mean from 5 individual experiments.

skeletal contractility within cells and thus manipulate the differentiation behavior of hMSCs.

Collectively, the use of a shape memory effect can be employed to enhance and rationally control differentiation-specific signaling, thereby guiding differentiation in engineered scaffolds. In addition, the effect of the cells gravity on their differentiation behavior is also firstly reported and employed. Therefore, this study not only provides one-of-a-kind shape memory hydrogels and methodologies for designing tissue engineering scaffolds, but also benefits the fundamental understanding of the differentiation behavior of stem cells in complex geometrical space. It is necessary to point out that we only provide a phenomenal interpretation for the observed heterogeneous differentiation of stem cells on a 3-D scaffold in this study. Nonetheless, the finding reported here hints to us that the different growth positions of cells in a $3 \mathrm{D}$ scaffold may influence their other physiological functions, and more experimental and theoretical work is needed to uncover this mechanism from materials science, cell biology as well as biophysics perspectives.

1. Nombela-Arrieta, C., Ritz, J. \& Silberstein, L. E. The elusive nature and function of mesenchymal stem cells. Nat. Rev. Mol. Cell Biol. 12, 126-131 (2011).

2. Jiang, Y. H. et al. Pluripotency of mesenchymal stem cells derived from adult marrow. Nature 418, 41-49 (2002).

3. Zhu, S., Wei, W. \& Ding, S. Chemical strategies for stem cell biology and regenerative medicine. Annu. Rev. Biomed. Eng. 13, 73-90 (2011).

4. Sun, Y., Chen, C. S. \& Fu, J. Forcing stem cells to behave: A biophysical perspective of the cellular microenvironment. Annu. Rev. Biophys. 41, 519-542 (2012).

5. Scadden, D. T. The stem-cell niche as an entity of action. Nature 441, 1075-1079 (2006).

6. Schugar, R. C., Robbins, P. D. \& Deasy, B. M. Small molecules in stem cell selfrenewal and differentiation. Gene Ther. 15, 126-135 (2008).

7. Higuchi, A., Ling, Q. D., Chang, Y., Hsu, S. T. \& Umezawa, A. Physical cues of biomaterials guide stem cell differentiation fate. Chem. Rev. 113, 3297-3328 (2013). 
8. Higuchi, A., Ling, Q. D., Ko, Y. A., Chang, Y. \& Umezawa, A. Biomaterials for the feeder-free culture of human embryonic stem cells and induced pluripotent stem cells. Chem. Rev. 111, 3021-3035 (2011).

9. Saha, K., Pollock, J. F., Schaffer, D. V. \& Healy, K. E. Designing synthetic materials to control stem cell phenotype. Curr. Opin. Chem. Biol. 11, 381-387 (2007).

10. McBeath, R., Pirone, D. M., Nelson, C. M., Bhadriraju, K. \& Chen, C. S. Cell shape, cytoskeletal tension, and RhoA regulate stem cell lineage commitment. Dev. Cell 6, 483-495 (2004).

11. Engler, A. J., Sen, S., Sweeney, H. L. \& Discher, D. E. Matrix elasticity directs stem cell lineage specification. Cell 126, 677-689 (2006).

12. Narayanan, K. et al. Extracellular matrix-mediated differentiation of human embryonic stem cells: differentiation to insulin-secreting beta cells. Tissue Eng. Part A 20, 424-433 (2014).

13. Trappmann, B. et al. Extracellular-matrix tethering regulates stem-cell fate. Nat. Mater. 11, 642-649 (2012).

14. Li, H., Wijekoon, A. \& Leipzig, N. D. 3D differentiation of neural stem cells in macroporous photopolymerizable hydrogel scaffolds. Plos One 7, e48824 (2012).

15. Yao, X., Peng, R. \& Ding, J. Cell-material interactions revealed via material techniques of surface patterning. Adv. Mater. 25, 5257-5286 (2013).

16. Wang, X., Ye, K., Li, Z. H., Yan, C. \& Ding, J. D. Adhesion, proliferation, and differentiation of mesenchymal stem cells on RGD nanopatterns of varied nanospacings. Organogenesis 9, 280-286 (2013).

17. McMurray, R. J. et al. Nanoscale surfaces for the long-term maintenance of mesenchymal stem cell phenotype and multipotency. Nat. Mater. 10, 637-644 (2011).

18. Kilian, K. A., Bugarija, B., Lahn, B. T. \& Mrksich, M. Geometric cues for directing the differentiation of mesenchymal stem cells. Proc. Natl. Acad. Sci. USA 107, 4872-4877 (2010)

19. Lutolf, M. P., Gilbert, P. M. \& Blau, H. M. Designing materials to direct stem-cell fate. Nature 462, 433-441 (2009)

20. Huebsch, N. et al. Harnessing traction-mediated manipulation of the cell/matrix interface to control stem-cell fate. Nat. Mater. 9, 518-526 (2010).

21. Kilian, K. A. \& Mrksich, M. Directing stem cell fate by controlling the affinity and density of ligand-receptor interactions at the biomaterials interface. Angew. Chem. Int. Ed. 51, 4891-4895 (2012).

22. Mei, Y. et al. Combinatorial development of biomaterials for clonal growth of human pluripotent stem cells. Nat. Mater. 9, 768-778 (2010).

23. Han, Y. J., Bai, T., Liu, Y., Zhai, X. Y. \& Liu, W. G. Zinc ion uniquely induced triple shape memory effect of dipole-dipole reinforced ultra-high strength hydrogels. Macromol. Rapid Commun. 33, 225-231 (2012).

24. Bai, T., Han, Y. J., Zhang, P., Wang, W. \& Liu, W. G. Zinc ion-triggered two-way macro-/microscopic shape changing and memory effects in high strength hydrogels with pre-programmed unilateral patterned surfaces. Soft Matter $\mathbf{8}$, 6846-6852 (2012)

25. Bai, T. et al. Construction of an ultrahigh strength hydrogel with excellent fatigue resistance based on strong dipole-dipole interaction. Soft Matter 7, 2825-2831 (2011).

26. Katz, A. K., Glusker, J. P., Beebe, S. A. \& Bock, C. W. Calcium ion coordination: A comparison with that of beryllium, magnesium, and zinc. J. Am. Chem. Soc. 118 , 5752-5763 (1996).

27. Discher, D. E., Mooney, D. J. \& Zandstra, P. W. Growth factors, matrices, and forces combine and control stem cells. Science 324, 1673-1677 (2009).

28. Zhang, R. et al. A thermoresponsive and chemically defined hydrogel for longterm culture of human embryonic stem cells. Nat. Commun. 4, 1335 (2013).

29. Khetan, S. et al. Degradation-mediated cellular traction directs stem cell fate in covalently crosslinked three-dimensional hydrogels. Nat. Mater. 12, 458-465 (2013).

30. Kwon, J. S. et al. In vivo osteogenic differentiation of human turbinate mesenchymal stem cells in an injectable in situ-forming hydrogel. Biomaterials 35, 5337-5346 (2014).
31. Annabi, N. et al. 25th anniversary article: rational design and applications of hydrogels in regenerative medicine. Adv. Mater. 26, 85-124 (2014).

32. Lee, J., Abdeen, A. A., Zhang, D. \& Kilian, K. A. Directing stem cell fate on hydrogel substrates by controlling cell geometry, matrix mechanics and adhesion ligand composition. Biomaterials 34, 8140-8148 (2013).

33. Oju, J. \& Eben, A. Regulation of stem cell fate in a three-dimensional micropatterned dual-crosslinked hydrogel system. Adv. Funct. Mater. 23, 4765-4775 (2013).

34. Dolatshahi-Pirouz, A. et al. A combinatorial cell-laden gel microarray for inducing osteogenic differentiation of human mesenchymal stem cells. Sci. Rep. 4, 3896 (2014).

35. Yoon, B. S. et al. Enhanced differentiation of human embryonic stem cells into cardiomyocytes by combining hanging drop culture and 5-azacytidine treatment. Differentiation 74, 149-159 (2006).

36. Nabavi, N., Khandani, A., Camirand, A. \& Harrison, R. E. Effects of microgravity on osteoclast bone resorption and osteoblast cytoskeletal organization and adhesion. Bone 49, 965-974 (2011).

37. Marie, P. J. et al. Osteobiology, strain, and microgravity: part I. studies at the cellular level. Calcif. Tissue Int. 67, 2-9 (2000).

38. Orr, A. W., Helmke, B. P., Blackman, B. R. \& Schwartz, M. A. Mechanisms of Mechanotransduction. Dev. Cell 10, 11-20 (2006).

39. Simard, E. et al. beta-Arrestin regulation of myosin light chain phosphorylation promotes AT1aR-mediated cell contraction and migration. Plos One 8, e80532 (2013).

40. Karamichos, D., Brown, R. A. \& Mudera, V. Collagen stiffness regulates cellular contraction and matrix remodeling gene expression. J. Biomed. Mater. Res. A 83, 887-894 (2007)

41. Formigli, L. et al. Sphingosine 1-phosphate induces cell contraction via calciumindependent/Rho-dependent pathways in undifferentiated skeletal muscle cells. J. Cell. Physiol. 198, 1-11 (2004).

\section{Acknowledgments}

This work is funded by the National Natural Science Foundation of China (Grants $51173129,21274105)$ and National Natural Science Funds for Distinguished Young Scholar (No. 51325305)

\section{Author contributions}

W.L. supervised the project. Y.H., T.B. and W.L. designed the study and wrote the paper. Y.H. and T.B. conducted the experiments. All authors read the paper and contributed to its final form. Y.H. and T.B. contributed equally to this work.

\section{Additional information}

Supplementary information accompanies this paper at http://www.nature.com/ scientificreports

Competing financial interests: The authors declare no competing financial interests How to cite this article: Han, Y., Bai, T. \& Liu, W. Controlled Heterogeneous Stem Cell Differentiation on a Shape Memory Hydrogel Surface. Sci. Rep. 4, 5815; DOI:10.1038/ srep05815 (2014).

This work is licensed under a Creative Commons Attribution-NonCommercialNoDerivs 4.0 International License. The images or other third party material in this article are included in the article's Creative Commons license, unless indicated otherwise in the credit line; if the material is not included under the Creative Commons license, users will need to obtain permission from the license holder in order to reproduce the material. To view a copy of this license, visit http:// creativecommons.org/licenses/by-nc-nd/4.0/ 